M.V. Yarmolenko

\title{
Intermetallics Disappearance Rates and Intrinsic Diffusivities Ratios Analysis in the $\mathrm{Cu}-\mathrm{Zn}$ and the Cu-Sn Systems
}

\author{
Kyiv National University of Technologies and Design, Faculty of Market, Information and Innovation Technologies, \\ Cherkasy, Ukraine, yarmolenko.mv@knutd.edu.ua
}

\begin{abstract}
Intermetallics disappearance rates and intrinsic diffusivities ratios in the $\mathrm{Cu}-\mathrm{Zn}$ system at temperature $400{ }^{\circ} \mathrm{Cand}$ in the $\mathrm{Cu}-\mathrm{Sn}$ system at temperatures from $190{ }^{\circ} \mathrm{C}$ to $250{ }^{\circ} \mathrm{C}$ are analyzed theoretically using literature experimental data. Diffusion activation energies and pre-exponential coefficients for the $\mathrm{Cu}-\mathrm{Sn}$ system are calculated combining literature experimental results.

Keywords: diffusion, intermetallics, phases formation kinetics, copper, zink, tin, Kirkendall-Frenkel porosity, Kirkendall shift.
\end{abstract}

Received 3 February 2021; Accepted 20 February 2021.

\section{Introduction}

A theoretical method to describe intermetallics disappearance rate in double multiphase systems was proposed in [1], and intermetallics disappearance rates in the Al-Cu system at temperatures from $175^{\circ} \mathrm{C}$ to $225^{\circ} \mathrm{C}$ were analyzed. Ref. 2 reported on another theoretical method of calculation the ratio of intrinsic diffusivities in double multiphase systems, and intrinsic diffusivities ratios of copper and aluminum in the $\mathrm{Al}-\mathrm{Cu}$ system were calculated at temperatures from $400{ }^{\circ} \mathrm{C}$ to $535^{\circ} \mathrm{C}$ and at temperatures less than $100^{\circ} \mathrm{C}$. Both methods are confirmed experimentally during the investigation the $\mathrm{Cu}-\mathrm{Zn}$ system at temperature $400{ }^{\circ} \mathrm{C}[5,8]$.

The soldered copper/tin based contacts are the weakest part of the chip that can be related to intermetallics and the Kirkendall-Frenkel porosity formation in the contact zone $[3,4,7]$. One of the most common reasons for chip failure is the soldered. The typical range of packaging and operation of the integrated circuits is from room temperature up to $250{ }^{\circ} \mathrm{C}$ [32]. Therefore, it is very important to analyze the intermetallics disappearance rates and intrinsic diffusivities ratios in the $\mathrm{Cu}-\mathrm{Sn}$ systems at temperatures from $190{ }^{\circ} \mathrm{C}$ to $250{ }^{\circ} \mathrm{C}$.

\section{Intermetallics disappearance rate analysis}

\section{I.1. The Zn-Cu system}

We didn't analyze intermetallics disappearance rate in the $\mathrm{Zn}-\mathrm{Cu}$ system $[5,8]$, so we can do it now. Three phases ( $\varepsilon$-brass, $\mathrm{Zn}_{5} \mathrm{Cu}, C_{1} \approx 0.83 ; \gamma$-brass, $\mathrm{Zn}_{8} \mathrm{Cu}_{5}$, $C_{2} \approx 0.62$; and $\beta$-brass, $\mathrm{ZnCu}, C_{3} \approx 0.5, C=C_{\mathrm{Zn}}$ ) are formed during diffusion. Reaction rates of phases formation at temperature $400{ }^{\circ} \mathrm{C}$ were measured: $K_{1} \approx 2025 \mu \mathrm{m}^{2} / \mathrm{h}, \quad K_{2} \approx 14400 \mu \mathrm{m}^{2} / \mathrm{h}, \quad K_{3} \approx 160 \mu \mathrm{m}^{2} / \mathrm{h}$, $K_{2} \approx 7 K_{1}, \quad K_{3} \approx 0,08 K_{1} \approx 0,011 K_{2}$. Initial $\mathrm{Zn}$ covering thickness was $X_{A} \approx 115 \mu \mathrm{m}$. Eqs.9 in [1] give:

$$
\begin{gathered}
K_{123}=\left(\sqrt{K_{1}}+\sqrt{K_{2}}+\sqrt{K_{3}}\right)^{2} \approx 31500 \mu \mathrm{m}^{2} / \mathrm{h}, \\
K_{12}=\left(\sqrt{K_{1}}+\sqrt{K_{2}}\right)^{2} \approx 27200 \mu \mathrm{m}^{2} / \mathrm{h}
\end{gathered}
$$

$\left(K_{3}<<K_{1}\right.$ and $\left.K_{3}<<K_{2}\right)$. Zinc disappearance time, $t_{0}$, at temperature $400^{\circ} \mathrm{C}$ can be estimated by Eq.11 [1]: 
Intermetallics Disappearance Rates and Intrinsic Diffusivities Ratios Analysis...

$$
t_{0}=\frac{X_{Z n}^{2}}{\left(C_{1} \sqrt{K_{1}}+C_{2} \sqrt{K_{2}}+C_{3} \sqrt{K_{3}}\right)^{2}} \approx 0.94 h \approx \frac{X_{Z n}^{2}}{C_{1}^{2} K_{12}} \approx 0.7 h .
$$

The value $t_{0}=0.94 h$ exactly corresponds with the experimentally obtained one [8] $t_{0}=0.92 \mathrm{~h}$ in planar samples. Phase 1 ( $\varepsilon$-brass, $\mathrm{Zn}_{5} \mathrm{Cu}$ ) disappearance time, $t_{l}$, can be estimated by Eq.13 [1]:

$$
t_{1}=\frac{X_{Z n}^{2}}{\left(C_{2} \sqrt{K_{2}^{(2 \text { phases })}}+C_{3} \sqrt{K_{3}^{(2 \text { phases })}}\right)^{2}} \approx 2 h \approx \frac{X_{Z n}^{2}}{C_{2}^{2} K_{12}} \approx 1.3 h
$$

Such values correspond to experimental result [8]: $t_{l}=1.64 h$ in cylindrical samples.

\section{I.2. The Cu-Sn system}

Two phases are formed in the $\mathrm{Cu}-\mathrm{Sn}$ system during isothermal annealing of $\mathrm{Cu} / \mathrm{Sn}$ samples at temperature $T=200{ }^{\circ} \mathrm{C} \quad[3,6,9]: \quad \varepsilon$-phase $\mathrm{Cu}_{3} \mathrm{Sn} \quad$ (phase 1, $\left.C_{l} \approx 3 / 4=0.753, \Delta C_{1}=0.012, C=C_{C u}[6]\right)$ and $\eta$-phase $\mathrm{Cu}_{6} \mathrm{Sn}_{5} \quad$ (phase 2, $C_{2} \approx 6 / 11=0.547, \quad \Delta C_{2}=0.021$, $\left.C=C_{C u}[6]\right)$, at temperature $T=210^{\circ} \mathrm{C}$ [10], and at temperature $\mathrm{T}=250^{\circ} \mathrm{C}$ ( $\mathrm{Sn}$ is liquid) [11]. Parabolic growth constants for the layer thicknesses were measured in [6], also the range of homogeneity of each phase were measured, and the values of the mutual diffusion coefficients for the $\mathrm{Cu}_{3} \mathrm{Sn}$ (phase 1) and $\mathrm{Cu}_{6} \mathrm{Sn}_{5}$ (phase 2) phases between $463 \mathrm{~K}$ and $493 \mathrm{~K}\left(190{ }^{\circ} \mathrm{C}\right.$ and $\left.220^{\circ} \mathrm{C}\right)$ were calculated too:

$$
\tilde{D}_{1}^{*}=1.43 \times 10^{-8} e^{-70.7 \mathrm{kJmol}^{-1} /(R T)} \mathrm{m}^{2} / \mathrm{s},
$$

$$
\tilde{D}_{2}^{*}=1.55 \times 10^{-8} e^{-64.9 k J m o l^{-1} /(R T)} m^{2} / s,
$$

and for phase 1 only $\left(\mathrm{Cu} / \mathrm{Cu}_{6} \mathrm{Sn}_{5}\right.$ sample) between $630 \mathrm{~K}$ and $677 \mathrm{~K}\left(357^{\circ} \mathrm{C}\right.$ and $\left.404^{\circ} \mathrm{C}\right)$ :

$$
\tilde{D}_{1}^{*(1 \text { phase })}=1.09 \times 10^{-6} e^{-78.2 \mathrm{kJmol}^{-1} /(R T)} \mathrm{m}^{2} / \mathrm{s} .
$$

We can calculate the mutual diffusion penetrability (or Wagner diffusivity [26]) of each phase, $D_{i}$, taking into account that $D_{i}=D_{i}{ }^{*} \cdot \Delta C_{i}$ and $\Delta C_{i}$ depends on temperature [6]:

$\Delta C_{l}=0.014\left(190^{\circ} \mathrm{C}\right)=0.018\left(210^{\circ} \mathrm{C}\right)=0.010\left(220^{\circ} \mathrm{C}\right)$, $\Delta C_{2}=0.023\left(190^{\circ} \mathrm{C}\right)=0.022\left(210^{\circ} \mathrm{C}\right)=0.017\left(220^{\circ} \mathrm{C}\right)$, $\Delta C_{l^{\text {lphase }}}=0.005\left(357^{\circ} \mathrm{C}\right)$. Mutual diffusion penetrabilities of phase 1can be calculated by Gibbs's method [12] or by "constant flux method" (Gurov's and Gusak's method) [13-23] or by other methods [26, 2830] (data are from [6] as an example):

$$
D_{1} \approx \frac{1}{2} C_{1}\left(1-C_{1}\right) K_{1} \approx \frac{3}{32} K_{1} \approx 2.5 \times 10^{-18} m^{2} / s \approx\left\{2.1 \times 10^{-18} m^{2} / s[6], T=190^{\circ} C\right\}
$$

Mutual diffusion penetrabilities of phase 1 and phase 2 can be calculated by "constant flux method" (data are from [9] as an example):

$$
\begin{gathered}
D_{1} \approx \frac{1}{2}\left(C_{1}\left(1-C_{1}\right) K_{1}+C_{2}\left(1-C_{1}\right) \sqrt{K_{1} K_{2}}\right) \approx \\
\approx \frac{3}{32} K_{1}+\frac{3}{44} \sqrt{K_{1} K_{2}} \approx 29.4 \times 10^{-18} m^{2} / s \approx\left\{19.4 \times 10^{-18} m^{2 / s[9]\}} ;\right. \\
D_{2} \approx \frac{1}{2}\left(C_{2}\left(1-C_{2}\right) K_{2}+C_{2}\left(1-C_{1}\right) \sqrt{K_{1} K_{2}}\right) \approx \\
\approx \frac{15}{121} K_{2}+\frac{3}{44} \sqrt{K_{1} K_{2}} \approx 53.6 \times 10^{-18} m^{2 / s} \approx\left\{56.4 \times 10^{-18} m^{2 / s[9]}\right\}
\end{gathered}
$$

so we can see a good agreement between applied methods (Table 1).

Initial $\mathrm{Cu}$ layer thickness [3] can be calculated using mass law

(Fig. 1):

$$
X_{C u}(t=0) \approx 5 \mu m+C_{1} X_{1}(t)+C_{2} X_{2}(t) \approx 12.4 \mu m .
$$

Copper layer disappearance time can be calculated using Eq. 1 (Fig. 2): 
Table 1

Comparison of diffusion penetrabilities calculated by different methods

\begin{tabular}{|c|c|c|c|c|c|c|c|c|c|c|}
\hline Authors & $T,{ }^{o} \mathrm{C}$ & $t, h$ & $\begin{array}{c}K_{l,} \\
x 10^{-18} \\
m^{2} / s\end{array}$ & $\begin{array}{c}K_{2} \\
x 10^{-18} \\
m^{2} / s\end{array}$ & $\begin{array}{l}\underline{K_{2}} \\
K_{1}\end{array}$ & $\begin{array}{c}D_{l}, \\
x 10^{-18} \\
m^{2} / s\end{array}$ & $\begin{array}{c}D_{2} \\
x 10^{-18} \\
\mathrm{~m}^{2} / \mathrm{s}\end{array}$ & $\frac{D_{2}}{D_{1}}$ & $\begin{array}{c}K_{I^{(1 p h a s e)}} \text {, } \\
x 10^{-18} \mathrm{~m}^{2} / \mathrm{s} \\
\mathrm{Cu} / \mathrm{Cu}_{6} \mathrm{Sn}_{5} \\
\text { couple }\end{array}$ & $\begin{array}{c}K_{2}^{(1 \text { lphase })}, \\
x 10^{-18} \mathrm{~m}^{2} / \mathrm{s} \\
\mathrm{Cu}_{3} \mathrm{Sn} / \mathrm{Sn} \\
\text { couple }\end{array}$ \\
\hline \multirow{5}{*}{$\begin{array}{l}\text { Onishi and } \\
\text { Fujibuchi } \\
\text { [6] }\end{array}$} & 190 & 400 & 27.2 & 88.2 & 3.2 & 2.1 & 18 & 8.6 & - & - \\
\hline & 200 & 400 & 38.4 & 112 & 2.9 & 2.6 & 23.5 & 9.0 & - & - \\
\hline & 210 & 400 & 52.1 & 142 & 2.7 & 5.7 & 34.4 & 6 & - & - \\
\hline & 220 & 400 & 74.1 & 204 & 2.75 & 4.5 & 37 & 8.2 & - & - \\
\hline & 357 & 16 & - & - & - & - & - & - & $\begin{array}{c}26900 \\
D_{I}^{(I \text { lphase })}= \\
1800\end{array}$ & - \\
\hline \multirow[t]{5}{*}{ This work } & 190 & - & - & - & - & 6 & 14.3 & 2.4 & - & - \\
\hline & 200 & - & - & - & - & 8.1 & 18.3 & 2.2 & - & - \\
\hline & 210 & - & - & - & - & 10.7 & 23.5 & 2.2 & - & - \\
\hline & 220 & - & - & - & - & 15.3 & 33.7 & 2.2 & - & - \\
\hline & 357 & - & - & - & - & - & - & - & $\begin{array}{c}D_{I^{(\text {lphase })}}= \\
1500\end{array}$ & - \\
\hline $\begin{array}{l}\text { Paul et al. } \\
\text { [9] }\end{array}$ & 200 & 225 & 100 & 332 & 3.3 & 19.4 & 56.4 & 2.9 & & - \\
\hline This work & - & - & - & - & - & 29.4 & 53.6 & 1.8 & - & - \\
\hline $\begin{array}{l}\text { Kumar et } \\
\text { al. [3] }\end{array}$ & 200 & 240 & 20 & 72 & 3.6 & 2.5 & 18.2 & 7.2 & 184 & - \\
\hline This work & - & - & - & - & - & 4.5 & 11.5 & 2.6 & $\begin{array}{c}D_{1}^{\text {(lphase) }}= \\
10.3 \approx D_{2}\end{array}$ & - \\
\hline $\begin{array}{l}\text { Liashenkoet } \\
\text { al. [11] }\end{array}$ & 250 & 8 & 820 & 6600 & 8.0 & - & - & - & - & 10270 \\
\hline This work & - & - & - & - & - & 236 & 975 & 4.1 & - & $\begin{array}{c}D_{2}{ }^{\text {(lphase) }}= \\
764 \approx D_{2}\end{array}$ \\
\hline
\end{tabular}

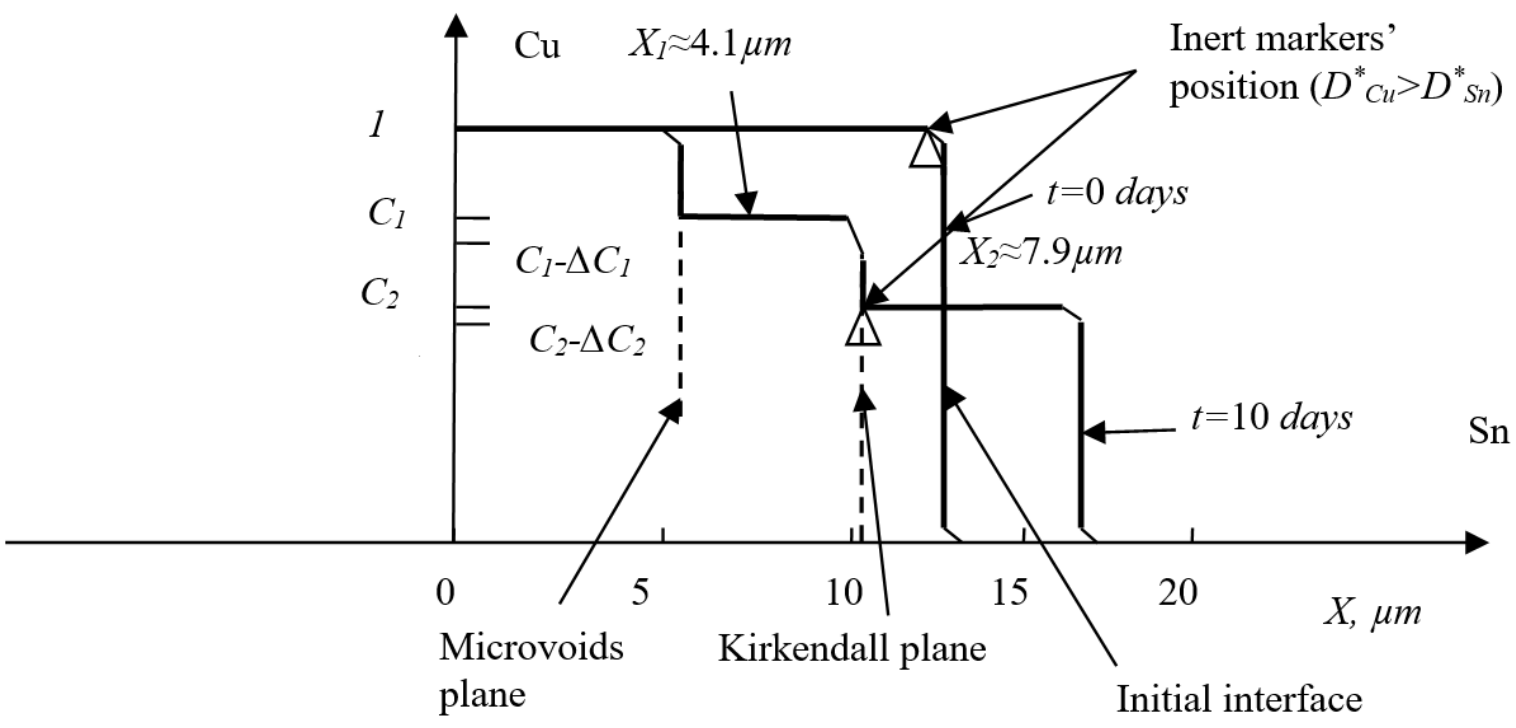

$X_{K} \approx 3 \mu m$

Fig. 1. Change of copper concentration profile during isothermal annealing of a $\mathrm{Cu}(99.9 \%) / \mathrm{Sn}$ couple at temperature $T=200^{\circ} \mathrm{C}$ within 10 days [3]: $D^{*}{ }_{C u}>D_{S n}^{*}, D^{*}{ }_{S n} \approx 0.31 D^{*}{ }_{C u} ; X_{1}<X_{2}$,

$X_{2} \approx 2 X_{1}, X_{K} \approx 3 \mu m ; C_{1}=3 / 4 ; C_{2}=6 / 11, C=C_{C u}$.

$t_{0}=\frac{X_{C u}^{2}}{\left(C_{1} \sqrt{K_{1}}+C_{2} \sqrt{K_{2}}\right)^{2}} \approx \frac{154 \times 10^{-12} \mathrm{~m}^{2}}{6.4 \times 10^{-17} \mathrm{~m}^{2} / \mathrm{s}} \approx 28$ days.

Phase 2 is formed between phase 1 and tin after disappearance of $\mathrm{Cu}$, phase 1 homogenization occur after this time, concentration decreases from $C_{1}$ to $C_{1}-\Delta C_{l}$, and 
Intermetallics Disappearance Rates and Intrinsic Diffusivities Ratios Analysis...

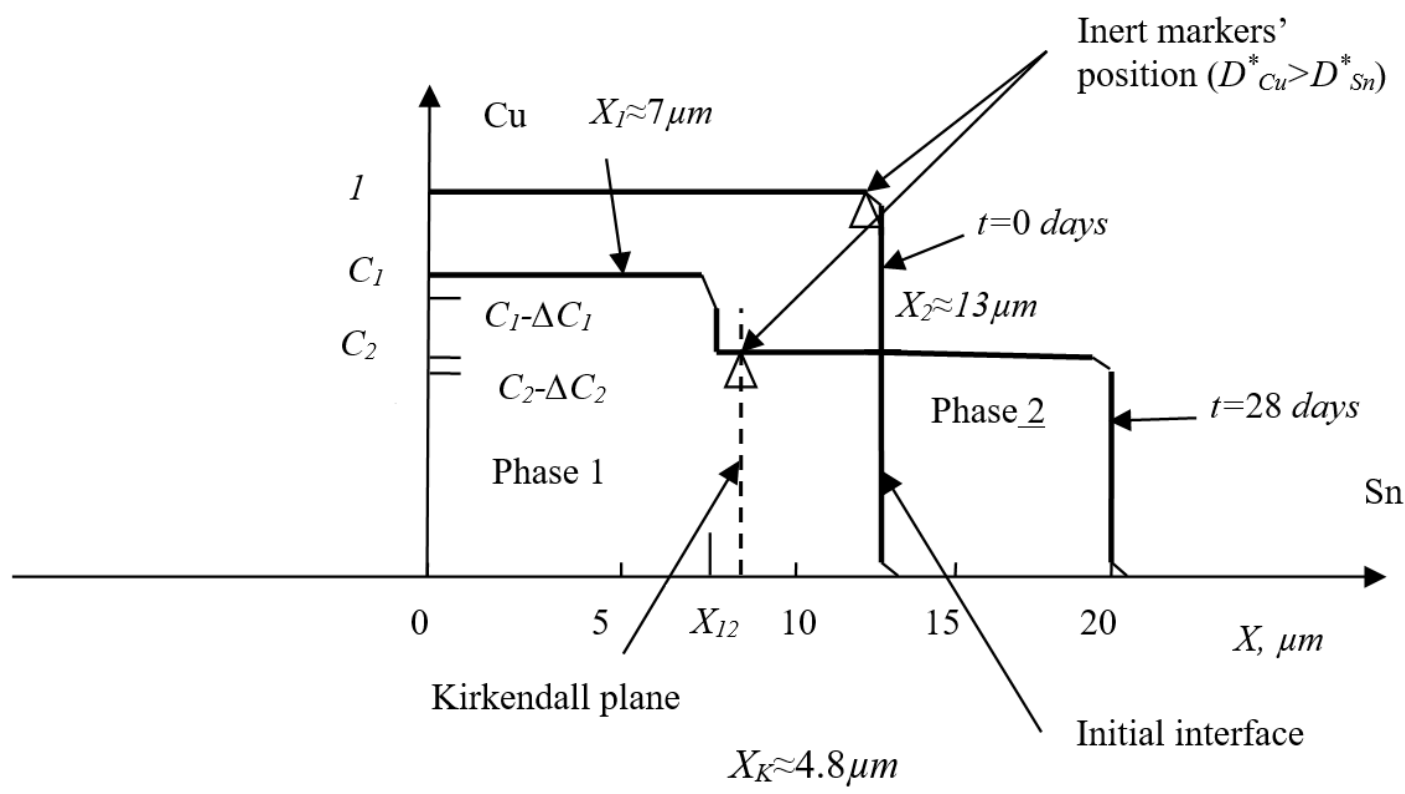

Fig. 2. Change of copper concentration profile during isothermal annealing at temperature $T=200^{\circ} \mathrm{C}$ within 28 days (calculation): $D^{*}{ }_{C u}>D^{*}{ }_{S n} ; X_{l}<X_{2} ; X_{2} \approx 2 X_{I} ; X_{K} \approx 4.8 \mu m ; C_{l}=3 / 4$;

$C_{2}=6 / 11, X_{12}$ is boundary between phase 1 and phase 2 .

boundary between phase 1 and phase $2, X_{12}$, moves to the left hand direction (see Fig. 2). The boundary moves to the left hand direction also after full homogenization of phase 1 . We can calculate assuming $C_{l}=3 / 4$ and $C_{2}=6 / 11$ and taking into account Eq. 6:

$$
D_{2}^{(1 \text { phase })} \approx \frac{C_{2}\left(C_{1}-C_{2}\right) K_{2}^{(1 \text { phase })}}{2 C_{1}} \approx \frac{9}{121} K_{2}^{(1 \text { phase })} \approx 764 \times 10^{-18} m^{2} / s \approx D_{2}^{(2 \text { phases })},
$$

as should be expected. Phase 1 layer disappearance time can be calculated using Eq. 2 (Fig. 3):

$$
t_{1}=\frac{X_{C u}^{2}}{C_{2}^{2} K_{2}^{(1 \text { phase })}} \approx 39 \text { days } .
$$

Phase 2 homogenization occur after this time, concentration decreases from $C_{2}$ to $C_{2}-\Delta C_{2}$, and boundary between phase 2 and $\mathrm{Sn}$ moves to the left hand direction.

\section{I.3. The Sn-Cu system}

We can analyze experimental results in the $\mathrm{Sn}-\mathrm{Cu}$ system described in [11] (Fig. 4).

Two phases are formed in the $\mathrm{Cu}$-Sn system during isothermal annealing of $\mathrm{Cu} / \mathrm{Sn}$ samples at temperature $T=250{ }^{\circ} \mathrm{C}$ during $t=8 \mathrm{~h}$ ( $\mathrm{Sn}$ is liquid): $X_{I}=3.97 \mu \mathrm{m}$, $X_{2}=12.75 \mu \mathrm{m} ; K_{1}=8.2 \times 10^{-16} \mathrm{~m}^{2} / \mathrm{s}, K_{2}=6.6 \times 10^{-15} \mathrm{~m}^{2} / \mathrm{s} ;$ $C_{2}=5 / 11, C_{l}=1 / 4, C=C_{S n}$. The thickness of tin layer over the $\mathrm{Cu}$ and $\mathrm{Cu}_{3} \mathrm{Sn}$ substrates varied from 20 to $200 \mu \mathrm{m}$. General phases formation rate can be calculated by Eqs. 9 in [1]:

$$
K_{12}=\left(\sqrt{K_{1}^{(2 \text { phases })}}+\sqrt{K_{2}^{(2 \text { phases })}}\right)^{2}=12065 \times 10^{-18} \mathrm{~m}^{2} / \mathrm{s} \approx K_{2}^{(1 \text { phase })}=10268 \times 10^{-18} \mathrm{~m}^{2} / \mathrm{s},
$$

so phase 2 growth rate, $K_{2}^{(1}{ }^{(1 \text { phase })}$, is approximately equal to initial general growth rate, $K_{12}$ (Table 1). The disappeared Sn thickness can be estimated: $X_{S n}(8 h)=C_{1} X_{1}(8 h)+C_{2} X_{2}(8 h) \approx 6.8 \mu m$. Tin layer disappearance time can be calculated using Eq.1:

$$
\begin{gathered}
t_{0}\left(X_{S n}=20 \mu m\right)=\frac{X_{S n}^{2}}{\left(C_{1} \sqrt{K_{1}}+C_{2} \sqrt{K_{2}}\right)^{2}} \approx 57 h \approx 2.4 \text { days } ; \\
t_{0}\left(X_{S n}=200 \mu m\right)=\frac{X_{S n}^{2}}{\left(C_{1} \sqrt{K_{1}}+C_{2} \sqrt{K_{2}}\right)^{2}} \approx 240 \text { days } \approx 8 \text { months } .
\end{gathered}
$$




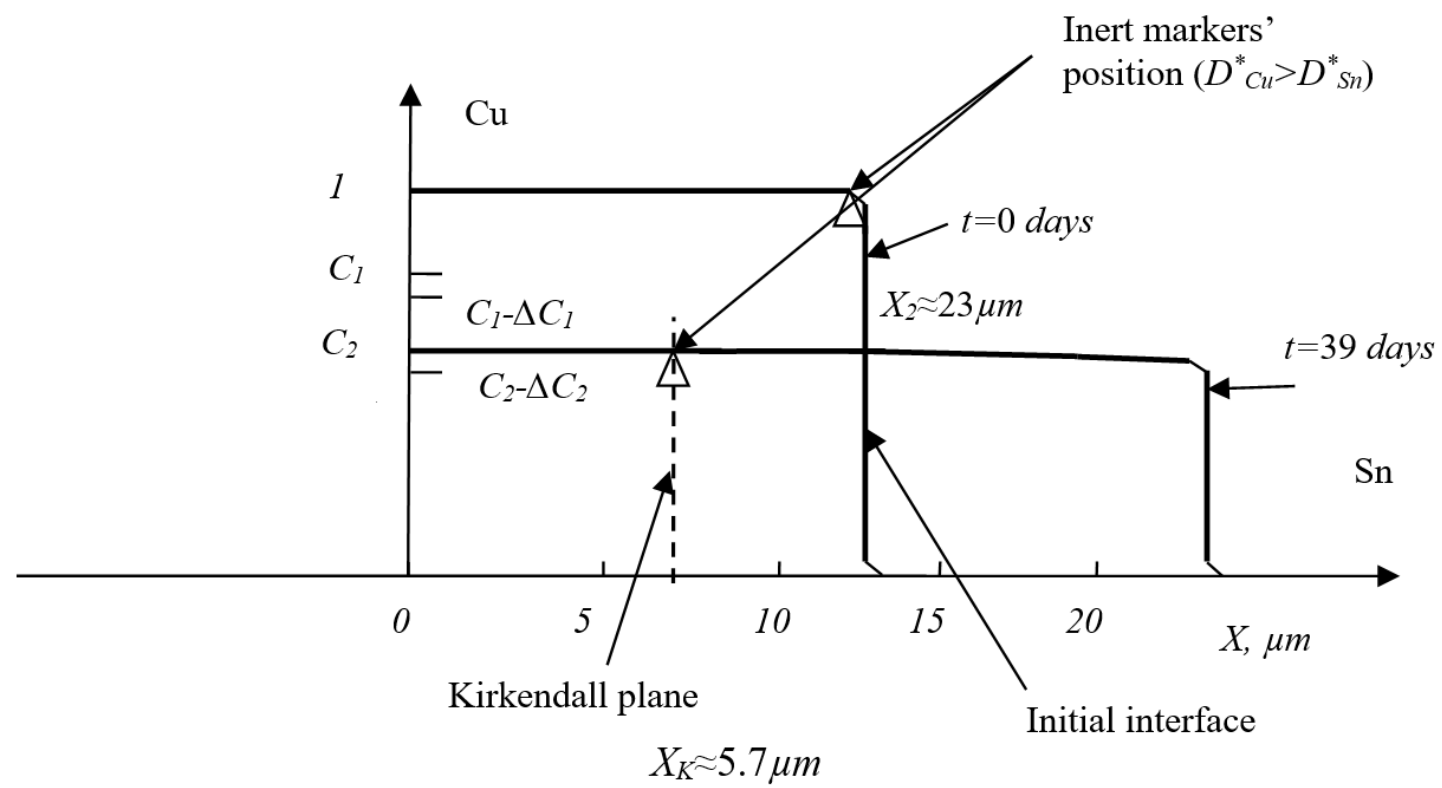

Fig. 3. Change of copper concentration profile during isothermal annealing at temperature $T=200{ }^{\circ} \mathrm{C}$ within 39 days (calculation): $D_{C u}^{*}>D_{S n}^{*} ; X_{2} \approx 23 \mu m ; X_{K} \approx 5.7 \mu m ; C_{2}=6 / 11 \approx 0.55$.

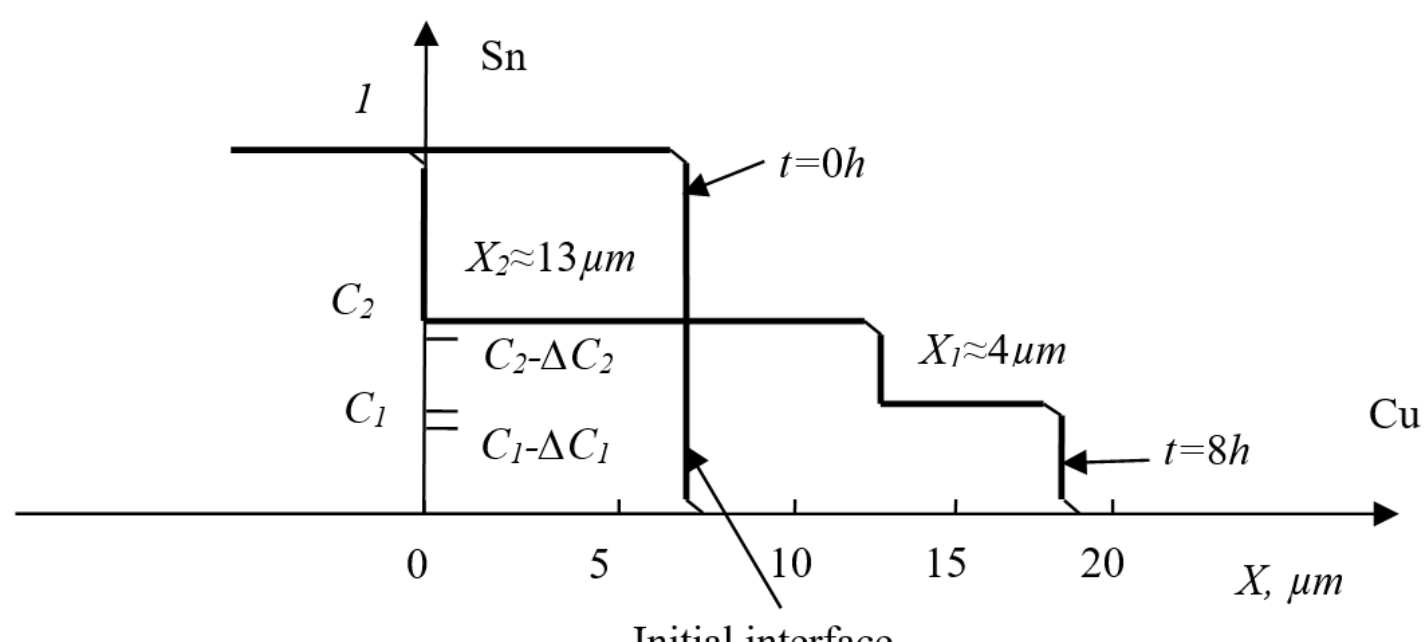

Initial interface

Fig. 4. Concentration profile of $\mathrm{Sn}$ change during isothermal annealing of a $\mathrm{Cu}(99.99 \%$ purity)/Sn couple at temperature $T=250^{\circ} \mathrm{C}$ within $8 h[11]: X_{2}=12.75 \mu \mathrm{m}, X_{1}=3.97 \mu \mathrm{m}, X_{2} \approx 3 X_{1}, C_{2}=5 / 11, C_{l}=1 / 4, C=C_{S n}$.

\section{Intrinsic Diffusivities Ratio Analysis}

\section{II.1. The $\mathrm{Zn-Cu}$ system}

We didn't analyze intrinsic diffusivities ratio in the $\mathrm{Zn}-\mathrm{Cu}$ system [5], so we can do it now. Three phases can formed: $\mathrm{Zn}_{5} \mathrm{Cu}$ (phase 1, $C_{1} \approx 0.83$ ), $\mathrm{Zn}_{8} \mathrm{Cu}_{5}$ (phase 2, $C_{2} \approx 0.62$ ), and $\mathrm{ZnCu}$ (phase 3, $C_{3} \approx 0.5, C=C_{Z n}$ ) (Fig. 5). Inert markers were in phase 2. Mutual diffusion coefficient in phase $2, D^{*}$, and Kirkendall shift, $X_{K}$, were measured at $T=400^{\circ} \mathrm{C}: D_{2}^{*}=9.1 \times 10^{-12} \mathrm{~m}^{2} / \mathrm{s}, \quad t=9 \mathrm{~h}$, $X_{K} \approx 150 \mu \mathrm{m}$. Ratio of intrinsic diffusivities can be calculated in such a way (Eq.16 in [2]):

$$
\frac{D_{C u}^{*}}{D_{Z n}^{*}} \approx \frac{\sum_{j=1}^{N} X_{j}-X_{K}\left(1-C_{i}\right) \sqrt{\pi}}{\sum_{j=1}^{N} X_{j}+C_{i} X_{K} \sqrt{\pi}} \approx 0.56<1,
$$

where $N=3$ is formed phases quantity, $X_{j}$ is phase $j$ 's thickness, $C_{i}$ is is the average concentration of $\mathrm{Zn}$ in phase $i$, or (Eq.15 in [2]):

$$
\frac{D_{C u}^{*}}{D_{Z n}^{*}}=\frac{\sqrt{D_{2}^{*}}-X_{K}\left(1-C_{2}\right) \sqrt{\pi}}{\sqrt{D_{2}^{*} t}+C_{2} X_{K} \sqrt{\pi}} \approx 0.62 \text {. }
$$

Experimentally obtained ratio $D^{*}{ }_{C u} / D_{Z n}^{*} \approx 0.6$ in $\alpha$-brass [24] and in $\gamma$-brass [25], so Eqs. 11 and 12 can be applied for other systems.

\section{II.2. The Cu-Sn system}

Another experiment was described in [3]. Phase 1 is formed between $\mathrm{Cu}$ and phase 2 during isothermal annealing at temperature $T=200{ }^{\circ} \mathrm{C}$ within 10 days: $X_{I} \approx$ $12.6 \mu \mathrm{m}, X_{K} \approx 4.9 \mu \mathrm{m} ; C_{l}=3 / 4, C_{2}=6 / 11$. The mutual diffusion penetrability of phase 1 can be calculated by 
Intermetallics Disappearance Rates and Intrinsic Diffusivities Ratios Analysis...

Eq. 6:

$$
\begin{gathered}
K_{1}^{(1 \text { phase })}=\frac{X_{1}^{2}}{t} \approx 184 \times 10^{-18} \mathrm{~m}^{2} / \mathrm{s} \approx \frac{2\left(1-C_{2}\right) D_{1}^{*} \Delta C_{1}}{\left(1-C_{1}\right)\left(C_{1}-C_{2}\right)}, \\
D_{1} \approx \frac{\left(1-C_{1}\right)\left(C_{1}-C_{2}\right)}{2\left(1-C_{2}\right)} \frac{X_{1}^{2}}{t} \approx 10.3 \times 10^{-18} \mathrm{~m}^{2} / \mathrm{s}, \\
D_{l}{ }^{*}=D_{l} / \Delta C_{l} \approx D_{I} / 0.012[6] \approx 860 \times 10^{-18} \mathrm{~m}^{2} / \mathrm{s} . \text { Ratio }
\end{gathered}
$$
$D^{*}{ }_{S n} / D^{*}{ }_{C u}$ in phase 1 can be calculated:

$$
\begin{gathered}
\frac{D_{S n}^{*}}{D_{C u}^{*}} \approx \frac{\sum_{j=1}^{N} X_{j}-X_{K}\left(1-C_{1}\right) \sqrt{\pi}}{\sum_{j=1}^{N} X_{j}+C_{1} X_{K} \sqrt{\pi}} \approx 0.74<1, \\
\mathrm{C}_{1}=3 / 4=\mathrm{C}_{\mathrm{Cu}}, \quad \mathrm{N}=2 .
\end{gathered}
$$

Experimentally determined value in phase 1 according [3] is : $D_{S n}^{*} \approx 0.4 \div 0.5 D^{*}{ }_{C u}$. Ref. [9] reported that $\mathrm{Cu}$ has a higher tracer diffusion coefficient than $\mathrm{Sn}$ in the $\mathrm{Cu}_{3} \mathrm{Sn}$ phase (phase 1) at temperatures from $225{ }^{\circ} \mathrm{C}$ to $350^{\circ} \mathrm{C}$. Ratio $D^{*}{ }_{S n} / D^{*}{ }_{C u}$ in phase 2 can also be calculated $\left(X_{K} \approx 3 \mu \mathrm{m}\right)$ :

$D_{2}{ }^{*}=D_{2} / \Delta C_{2} \approx 18.2 \times 10^{-18} \mathrm{~m}^{2} \mathrm{~s}^{-1} / 0.021[6] \approx 870 \times 10^{-18} \mathrm{~m}^{2} / \mathrm{s}$,

$$
\frac{D_{S n}^{*}}{D_{C u}^{*}} \approx \frac{\sum_{j=1}^{N} X_{j}-X_{K}\left(1-C_{2}\right) \sqrt{\pi}}{\sum_{j=1}^{N} X_{j}+C_{2} X_{K} \sqrt{\pi}} \approx 0.8<1,
$$

$$
C_{2}=6 / 11=C_{C u}, \quad N=2 .
$$

Experimentally determined value is as follows [3]: $D^{*}{ }_{S n} \approx 0.31 D^{*}{ }_{C u}$ in phase 2. Otherwise, according $[6,9]$ the diffusivity of $\mathrm{Sn}$ is higher than the diffusivity of $\mathrm{Cu}$ at temperatures $150{ }^{\circ} \mathrm{C} \div 220^{\circ} \mathrm{C}$, and markers moves toward $\mathrm{Sn}$ side in phase 2. More precise estimation of ratio $D_{S n}^{*} / D^{*}$ in phase 2 needs careful experiments under hydrostatic pressure of Argon gas $(\approx 10 \mathrm{MPa})[5$, $8]$ or under hot isostatic pressing ( $\mathrm{p} \approx 100 \mathrm{MPa}$, Argon) to decrease Kirkendal-Frenkel porosity formation [33].

\section{III.Diffusion activation energy calculation in the $\mathrm{Cu}-\mathrm{Sn}$ system}

We can calculate the diffusion activation energies and the pre-exponential factors combining experimental results at the temperature $T_{2}=357^{\circ} \mathrm{C} \quad[6]$ and experimental results at the temperature $T_{1}=200{ }^{\circ} \mathrm{C}$ [3] and combining experimental results at the temperature $T_{2}=250^{\circ} \mathrm{C}[11]$ and experimental results $[3,9,6]$ at the temperature $T_{l}=200{ }^{\circ} \mathrm{C}$ (Table 2):

\begin{tabular}{|c|c|c|c|c|c|c|c|}
\hline & & $\begin{array}{c}\text { Onishi and } \\
\text { Fujibuchi } \\
\text { [6] }\end{array}$ & $\begin{array}{l}\text { This } \\
\text { work }\end{array}$ & $\begin{array}{c}\text { Paul et al. } \\
\text { [9] }\end{array}$ & This work & $\begin{array}{c}\text { Kumar } e t \\
a l . ~[3]\end{array}$ & This work \\
\hline \multirow[t]{2}{*}{$Q_{i,} \mathrm{~kJ} / \mathrm{mol}$} & $1=\mathrm{Cu}_{3} \mathrm{Sn}$ & 70.7 & 124 & 73.8 & 85.7 & 38.7 & 39.0 \\
\hline & $2=\mathrm{Cu}_{6} \mathrm{Sn}_{5}$ & 64.8 & 142 & 81 & 119.5 & 47.3 & 43.4 \\
\hline \multirow[t]{2}{*}{$D_{0 i}, m^{2} / s$} & $1=\mathrm{Cu}_{3} \mathrm{Sn}$ & $1.43 \times 10^{-8}$ & $3.77 \times 10^{-}$ & $2.7 \times 10^{-9}$ & $8.7 \times 10^{-8}$ & - & $9 \times 10^{-6}$ \\
\hline & $2=\mathrm{Cu}_{6} \mathrm{Sn}_{5}$ & $1.55 \times 10^{-8}$ & 4 & $5.6 \times 10^{-8}$ & $8.7 \times 10^{-4}$ & - & $7 \times 10^{-5}$ \\
\hline$Q_{2} / Q_{1}$ & $\mathrm{Cu} / \mathrm{Sn}$ couple & 0.92 & $\begin{array}{c}8.2 \times 10^{-2} \\
1.15\end{array}$ & 1.1 & 1.4 & 1.22 & 1.11 \\
\hline $\begin{array}{l}Q_{\left.I^{(I p h a s e}\right)}, \\
\mathrm{kJ} / \mathrm{mol} \\
\left(T_{2}=357^{\circ} \mathrm{C}\right)\end{array}$ & $\begin{array}{c}1=\mathrm{Cu}_{3} \mathrm{Sn} \\
\left(\mathrm{Cu} / \mathrm{Cu}_{6} \mathrm{Sn}_{5}\right. \\
\text { couple })\end{array}$ & 78.2 & 78.6 & - & - & - & - \\
\hline$D_{01}, m^{2} / s$ & & $1.09 \times 10^{-6}$ & $5 \times 10^{-9}$ & - & - & - & - \\
\hline
\end{tabular}

$$
Q_{i}=\frac{R T_{1} T_{2}}{T_{2}-T_{1}} \ln \left(\frac{D_{i}\left(T_{2}\right)}{D_{i}\left(T_{1}\right)}\right),
$$

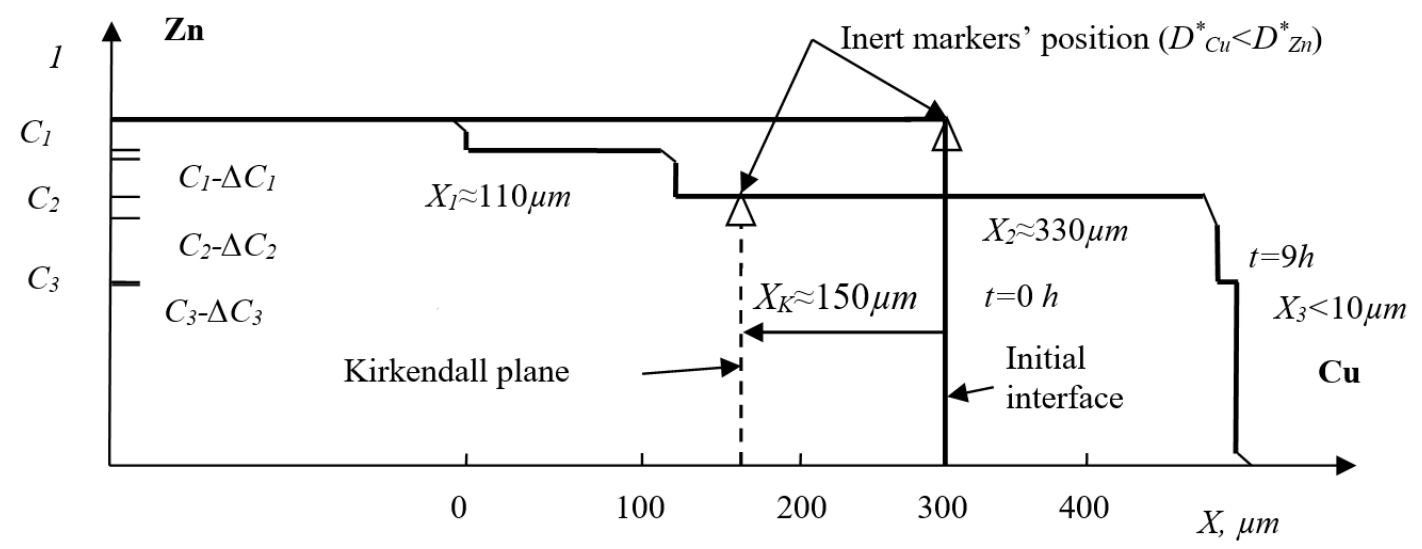

Fig. 5. Concentration profile of $\mathrm{Zn}$ change during isothermal annealing at temperature $T=400^{\circ} \mathrm{C}$ within $9 h$ [5]: $D^{*}{ }_{Z n}>D_{C u}^{*} ; X_{1}<X_{2}, X_{2}=3 X_{1}, X_{3} \approx 0 ; C_{1}=5 / 6, C_{2}=8 / 13, C_{3}=1 / 2, C=C_{Z n}$.

Table 2

Comparison of the diffusion activation energies and the pre-exponential factors 


$$
\begin{aligned}
& D_{0 i}=D_{i}\left(T_{1}\right) e^{Q_{i} /\left(R T_{1}\right)}=D_{i}\left(T_{2}\right) e^{Q_{i} /\left(R T_{2}\right)} . \\
& Q_{i}=-\frac{5 \sum_{j=1}^{5}\left(\frac{1000}{R T_{j}} \ln D_{i}\left(T_{j}\right)\right)-\sum_{j=1}^{5} \ln D_{i}\left(T_{j}\right) \sum_{j=1}^{5} \frac{1000}{R T_{j}}}{\left.5 \sum_{j=1}^{5}\left(\frac{1000}{R T_{j}}\right)^{2}-\left(\sum_{j=1}^{5} \frac{1000}{R T_{j}}\right)^{2} / \mathrm{mol}\right]},
\end{aligned}
$$

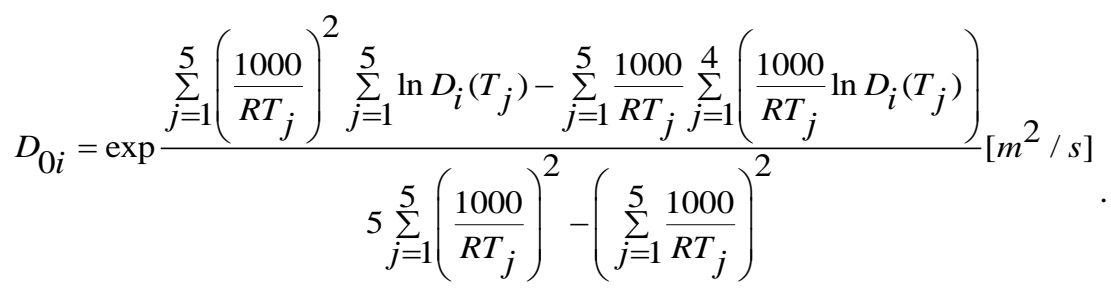

We can use five points $\left(T_{1}=190{ }^{\circ} \mathrm{C}, T_{2}=200{ }^{\circ} \mathrm{C}\right.$, $T_{3}=210^{\circ} \mathrm{C}, T_{4}=220^{\circ} \mathrm{C}$ [6], and $T_{5}=250^{\circ} \mathrm{C}$ [11]) for

Eqs.17 and 18 give Eqs.16 only for two points.

The data points from the $\mathrm{Sn} / \mathrm{Cu}$ couple are in good agreement with thedata from the incremental couples $\mathrm{Cu} / \mathrm{Cu}_{6} \mathrm{Sn}_{5}$ and $\mathrm{Cu}_{3} \mathrm{Sn} / \mathrm{Sn}$ [9]. We can see a good agreement between calculations and results described in $[3,6]$ for one phase $1\left(\mathrm{Cu} / \mathrm{Cu}_{6} \mathrm{Sn}_{5}\right.$ couple $)$. Really, the ratio $Q_{2} / Q_{1}$ should be less than 1 if $D_{01} \approx D_{02}$, as reported in [6].

The Sn diffusion coefficients in a concentrated solution (8 at. \% Sn) are several times greater than $\mathrm{Sn}$ diffusion coefficients in dilute solution $(2$ at. \% Sn) at temperatures from $500{ }^{\circ} \mathrm{C}$ to $650{ }^{\circ} \mathrm{C}$ [31], and diffusion activation energy of $\mathrm{Sn}$ vary from $89 \mathrm{~kJ} / \mathrm{mol}$ to $187 \mathrm{~kJ} / \mathrm{mol}$ (isotope data on Sn diffusion in a pure copper), therefore our calculated values should be correct.

\section{Conclusions}

The Znatoms have higher intrinsic diffusivities than the Cuatoms at temperature $400{ }^{\circ} \mathrm{C}$ in $\gamma$-brass $\mathrm{Cu}_{5} \mathrm{Zn}_{8}$, vacancies can disappear near dislocations at $\mathrm{Zn}$ side (sinks) and appear near dislocations at $\mathrm{Cu}$ side (sources), dislocations can climb, and the Kirkendall plane shifts toward $\mathrm{Zn}$ side. Calculated ratio $D^{*}{ }_{\mathrm{Cu}} / D_{\mathrm{Zn}}^{*} \approx 0.6$ in $\gamma$-brass $\mathrm{Cu}_{5} \mathrm{Zn}_{8}$ is the same as experimentally obtained at temperature $400{ }^{\circ} \mathrm{C}$.

The Cuatoms have higher intrinsic diffusivities than the Snatoms at temperature $200{ }^{\circ} \mathrm{C}$ in $\varepsilon$-phase $\mathrm{Cu}_{3} \mathrm{Sn}$, vacancies can disappear near dislocations at $\mathrm{Cu}$ side (sinks) and appear near dislocations at Sn side (sources), dislocations can climb, and the Kirkendall plane shifts toward $\mathrm{Cu}$ side. Calculated ratio $D^{*}{ }_{S n} / D^{*} \mathrm{Cu} \approx 0.7$ in $\varepsilon$-phase $\mathrm{Cu}_{3} \mathrm{Sn}$ is approximately equal to experimentally obtained at temperature $200{ }^{\circ} \mathrm{C}$.

Yarmolenko M.V. - Ph.D., Associate Professor, Head of the Department of Information and Computer Technologies and Fundamental Disciplines.

[1] M.V. Yarmolenko, Physics and Chemistry of Solid State, 21(2), 294 (https://doi.org/10.15330/pcss.21.2.294-299).

[2] M.V. Yarmolenko, Physics and Chemistry of Solid State, 21(4), 720 (2020) (DOI: 10.15330/pcss.21.4.720$\underline{726})$.

[3] S. Kumar, C.A. Handwerker, and M.A. Dayananda, JPEDAV 32, 309 (2011) (10.1007/s11669-011-990791547-7037).

[4] K.N. Tu, Electronic Thin-Film Reliability (Cambridge University Press, 2010) (https://www.amazon.com/Electronic-Thin-Film-Reliability-King-Ning-Tu/dp/0521516137).

[5] V.V. Bogdanov, L.N. Paritskaya, and M.V. Yarmolenko, Metallofizika 12(5), 98 (1990) (https://www.researchgate.net/publication/328789333_Effect_of_Internal_Stresses_on_Diffusion_Phase_Gro wth in Cylindrical_Specimens).

[6] M. Onishi and M. Fujibuchi, Trans. Jpn. Inst. Met. 16, 539 (1975) (https://www.jstage.jst.go.jp/article/matertrans1960/16/9/16 9 539/_pdf/-char/en).

[7] Y. Wang, Y. Liu, M. Li, K.N. Tu, L. Xu, Interconnect Quality and Reliability of 3D Packaging. In: Li Y., Goyal D. (eds) 3D Microelectronic Packaging. Springer Series in Advanced Microelectronics, 64. (Springer, Singapore, 2021) (https://doi.org/10.1007/978-981-15-7090-2_17).

[8] V.V. Bogdanov, A.M. Gusak, L.N. Paritskaya, and M.V. Yarmolenko, Metallofizika 12(3), 60 (1990) (https://www.researchgate.net/publication/328769306_Osobennosti_diffuzionnogo_rosta_faz_v_obraztsakh_ts ilindricheskoy formy). 
[9] A. Paul, C. Ghosh, and W.J. Boettinger, Metallurgical and materials transactions A, 42A, 952 (2011) (DOI: 10.1007/s11661-010-0592-9).

[10] V.V. Morozovych, A.R. Honda, Yu.O. Lyashenko, Ya.D. Korol, O.Yu. Liashenko, C. Cserhati, and A.M. Gusak, Metallofiz. Noveishie Tekhnol. 40(12), 1649 (2018) (DOI: 10.15407/mfint.40.12.1649).

[11] O. Liashenko, A.M. Gusak, F. Hodaj, J Mater Sci: Mater Electron, 25(10), 4664 (2014) (DOI 10.1007/s10854014-2221-7).

[12] G.B. Gibbs, Journal of Nuclear Materials 20(3), 303 (1966) (https://doi.org/10.1016/0022-3115(66)90042-0).

[13] M.V. Yarmolenko, A.M. Gusak, and K.P. Gurov, Journal of Engineering Physics and Thermophysics 65,876 (1993) (https://doi.org/10.1007/BF00862930).

[14] M.V. Yarmolenko, Defect and Diffusion Forum 143-147, 1567 (1997) (https://doi.org/10.4028/www.scientific.net/DDF.143-147.1567).

[15] M.V. Yarmolenko, Metallofiz. Noveishie Tekhnol. 40(9), 1201 (2018) (DOI: 10.15407/mfint.40.09.1201).

[16] M.V. Yarmolenko, AIP Advances, 8, 095202 (2018) (https://doi.org/10.1063/1.5041728).

[17] A.M. Gusak and M.V. Yarmolenko, J. Appl. Phys. 73(10), 4881 (1993) (https://doi.org/10.1063/1.353805)

[18] M.V. Yarmolenko, Proceedings of an International Conference on Solid - Solid Phase Transformations, 1177 (1994)

(https://www.researchgate.net/publication/329069886_deviation_from_parabolic growth_of_phase_layers_in cylindrical_and_spherical_samples_curvature_and_internal_stress_influence).

[19] M.V. Yarmolenko, Russian Metallurgy 3, 187 (1990)

(https://www.researchgate.net/publication/328698369_Stress_in_spherical_samples_with_mutual_diffusion).

[20] M.V. Yarmolenko, Defect and Diffusion Forum 143-147, 509 (1997) (https://doi.org/10.4028/www.scientific.net/DDF.143-147.509).

[21] A.M. Gusak, O.Yu. Liashenko, F. Hodaj, Fizika i Khimia obrabotki materialov 2, 37 (2018) (DOI:10.30791/0015-3214-2018-2-37-47).

[22] M.V. Yarmolenko, Metallofiz. Noveishie Tekhnol. 42(11), 1537 (2020) (DOI: 10.15407/mfint.42.11.1537).

[23] A.M. Gusak, Metallofiz. Noveishie Tekhnol. 42(10), 1335 (2020) (DOI: 10.15407/mfint.42.10.1335).

[24] A.D. Smigelkas and E.O. Kirkendall, Trans. AIME 171, 130 (1947).

[25] Ya.Ye. Geguzin,Yu. Kaganovskii, L.M. Paritskaya, and V.I. Solunskiy, Phys. Met. Metall. 47(4), 127 (1980) (https://www.researchgate.net/publication/292667643_KINETICS_OF THE_MOTION_OF_THE_INTERFA CE_DURING_MUTUAL_DIFFUSION_IN_A_TWO-COMPONENT_SYSTEM)

[26] C. Wagner, $\quad$ Acta $\quad$ Metall. $\quad 17(2), \quad 99$

(https://www.sciencedirect.com/science/article/abs/pii/000161606990131X?via\%3Dihub).

[27] B. Camin and L. Hansen, Metals, 10(8) 1034 (2020) (DOI:10.3390/met10081034).

[28] F.J.J. Van Loo, Acta Metall. 18(10), 1107 (1970) (https://www.sciencedirect.com/science/article/abs/pii/000161607090009X).

[29] G. Guy, Scripta Metall. 5(4), 279 (1971) (https://www.sciencedirect.com/science/article/abs/pii/0036974871901943).

[30] M.A. Dayananda, Defect Diffus. Forum, 95-98, 521 (1993) (https://www.scientific.net/DDF.95-98.521).

[31] V. Nikulkina, A.O. Rodin, B. Bokstein, Materials Letters 257, 1265252019 (2019) (DOI: 10.1016/j.matlet.2019.126525).

[32] A.M. Gusak, K.N. Tu, C. Chen, Scripta Materialia 179, 45 (2020). (https://doi.org/10.1016/j.scriptamat.2020.01.005).

[33] A. Epishin, B. Camin, L. Hansen, J. Schmidt, Materials Science Forum 1016, 102 (2021) (DOI:10.4028/www.scientific.net/MSF.1016.102).

\section{М.В. Ярмоленко}

\section{Аналіз кінетики зникнення інтерметалідів та відношення внутрішніх коефіціснтів дифузії у системах $\mathrm{Cu}-\mathrm{Zn}$ та $\mathrm{Cu}-\mathrm{Sn}$}

Київський національний університет технологій та дизайну, факультет ринку, інформаційних та інноваційних технологій, Черкаси, Україна, yarmolenko.mv@knutd.edu.иа

Теоретично проаналізована кінетика зникнення інтерметалідів у системіCu-Zn при температурі $400{ }^{\circ} \mathrm{C}$ та у системі $\mathrm{Cu}-\mathrm{Sn}$ при температурах від $190^{\circ} \mathrm{C}$ до $250^{\circ} \mathrm{C}$. Обчислено відношення внутрішніх коефіцієнтів дифузії у системі $\mathrm{Cu}-\mathrm{Zn}$ при температурі $400{ }^{\circ} \mathrm{C}$ та у системі $\mathrm{Cu}-\mathrm{Sn}$ при температурі $200{ }^{\circ} \mathrm{C}$. Знайдено також енергію активації дифузії та передекспонентні множники для системи Cu-Sn. Для аналізу були використані літературні експериментальні дані.

Ключові слова:дифузія, інтерметаліди, кінетика утворення фаз, мідь, цинк, олово, пористість Кіркендалла-Френкеля, зсув Кіркендалла. 